The median reported current daily dose of prednisolone was $15 \mathrm{mg}$ and median treatment duration was 5.3 years at the time of survey completion. Females were more likely to stay on GCs for longer than males (5.4 vs 4.7 years, $p=0.046$ ).

Table 1. Mean severity and frequency for each adverse effect.

\begin{tabular}{lccccc}
\hline & $\begin{array}{c}\text { All respondents } \\
(\mathbf{n}=\mathbf{1 2 2})\end{array}$ & $\begin{array}{c}\text { Male } \\
(\mathbf{n}=\mathbf{2 6})\end{array}$ & $\begin{array}{c}\text { Female } \\
(\mathbf{n}=\mathbf{9 6})\end{array}$ & $\begin{array}{c}<60 \text { years } \\
(\mathbf{n}=\mathbf{8 9})\end{array}$ & $\begin{array}{c}\mathbf{2 6 0} \text { years } \\
(\mathbf{n}=\mathbf{3 3})\end{array}$ \\
\hline Moon face & $3.3(1.9)$ & $2(2.1)^{\star}$ & $3.6(1.7)^{\star}$ & $3.5(1.8)$ & $2.7(2)$ \\
Weight gain & $3.4(1.7)$ & $3.1(1.6)$ & $3.4(1.8)$ & $3.5(1.7)$ & $3.1(1.9)$ \\
Acne & $1.1(1.5)$ & $1(1.5)$ & $1.2(1.6)$ & $1.3(1.6)$ & $0.7(1.2)$ \\
Hair loss & $2(1.8)$ & $0.8(1.4)^{\star}$ & $2.4(1.8)^{\star}$ & $2.2(1.8)^{\star *}$ & $1.5(1.9)^{\star *}$ \\
Facial hair & $2.1(1.8)$ & $0.7(1.3)^{\star}$ & $2.5(1.8)^{\star}$ & $2.3(1.8)$ & $1.7(1.8)$ \\
Thin skin & $2.2(1.9)$ & $1.8(2)$ & $2.3(1.8)$ & $2.2(1.9)$ & $2.3(1.9)$ \\
Bruising & $2.2(1.7)$ & $2.1(1.9)$ & $2.2(1.7)$ & $2.2(1.7)$ & $2(1.9)$ \\
Stretch marks & $1.3(1.9)$ & $0.9(1.5)$ & $1.4(1.8)$ & $1.5(1.9)^{\star *}$ & $0.6(1.2)^{\star *}$ \\
Nausea & $1.4(1.7)$ & $1.6(1.8)$ & $1.4(1.6)$ & $1.5(1.6)$ & $1.2(1.7)$ \\
Heartburn & $1.9(1.8)$ & $1.8(1.9)$ & $1.9(1.8)$ & $2(1.8)$ & $1.5(1.8)$ \\
Hunger & $2.8(1.7)$ & $2.2(1.9)^{\star}$ & $3(1.6)^{\star}$ & $3(1.6)$ & $2.4(2)$ \\
Mood swings & $2.7(1.6)$ & $2.1(1.8)$ & $2.7(1.5)$ & $2.8(1.6)$ & $2.6(1.6)$ \\
Sleep disturbances & $3.1(1.5)$ & $2.9(1.7)$ & $3.2(1.4)$ & $3.2(1.5)$ & $3(1.5)$ \\
Cataracts & $0.6(1.3)$ & $1.1(1.8)$ & $0.5(1.1)$ & $0.5(1.2)$ & $1(1.6)$ \\
Glaucoma & $0.4(1.1)$ & $0.6(1.5)$ & $0.3(0.9)$ & $0.4(1)$ & $0.4(1)$ \\
Hypertension & $1.6(1.7)$ & $1.8(1.7)$ & $1.5(1.7)$ & $1.5(1.7)$ & $1.8(1.8)$ \\
Diabetes & $0.8(1.6)$ & $1(1.6)$ & $0.8(1.6)$ & $0.7(1.5)$ & $1(1.8)$ \\
Water retention & $2.2(1.7)$ & $1.7(1.6)$ & $2.3(1.8)$ & $2.3(1.8)$ & $1.9(1.6)$ \\
Infections & $2.2(1.7)$ & $2.2(1.8)$ & $2.2(1.7)$ & $2.2(1.7)$ & $2.1(1.7)$ \\
Fractures & $0.5(1.1)$ & $0.5(1.2)$ & $0.5(1.1)$ & $0.5(1.6)$ & $0.5(1.1)$
\end{tabular}

Mean severity on a Likert scale (0-5), mean (SD); ${ }^{*} \mathrm{p}<0.05$, male vs female; ${ }^{* *} \mathrm{p}<0.05,<60$ years vs $\geq 60$ years.

Frequency (number of patients reporting adverse effect as present), heatmap: White (0-33\%), light grey (33-66\%), dark grey $(66-100 \%)$

The mean number of reported AEs to GC treatment per patient was 12 (SD 5). Female respondents reported a greater number of AEs compared to males (12.6 vs 10.1, $\mathrm{p}=0.019)$. The three most commonly experienced AEs were: sleep disturbance (93\%), mood swings (86\%) and weight gain (86\%). Only $54 \%$ of respondents agreed that the information given about the potential risks of GC treatment was adequate. Mean severity and frequency of AEs is represented in table 1.

Conclusion: Adverse effects are common, and those rated more severe by patients were physical and behavioural (such as weight gain or sleep disturbance). Female patients reported more severe AEs compared to men.

References:

[1] Van Der Goes MC, Jacobs JWG, Boers M, Andrews T, Blom-Bakkers MAM Buttgereit $F$, et al. Patient and rheumatologist perspectives on glucocorticoids: An exercise to improve the implementation of the European League Against Rheumatism (EULAR) recommendations on the management of systemic glucocorticoid therapy in rheumatic diseases. Ann Rheum Dis. 2010 Jun;69(6):1015-21.

Disclosure of Interests: None declared

DOI: 10.1136/annrheumdis-2020-eular.1119

\section{SAT0632-HPR PATIENTS VIEW ON CONSULTATION IN OUTPATIENT CLINIC - FROM SATISFACTION TO DEMAND OF GUIDANCE INTO MORE SELF-MANAGEMENT}

I. Lund ${ }^{1}$, A. Hansen ${ }^{1}$, B. Stampe ${ }^{1}$, R. Cordtz ${ }^{1,2}$, L. Dreyer ${ }^{1,3} .{ }^{1}$ Copenhagen University Hospital Gentofte, Center for Rheumatology and Spine Diseases, Copenhagen, Denmark; ${ }^{2}$ Aalborg University Hospital, Department of Rheumatology, Aalborg, Denmark; ${ }^{3}$ Aalborg University Hospital, Department of Rheumatology, Aalborg, Denmark

Background: Patients with rheumatic diseases, routinely consult the outpatient clinic 1-2 times a year to see their rheumatologist. Many of the patients also attend nurse-led consultations to receive instructions, regarding treatment in association to their intravenous treatment, or DMARD dispensing in the clinic. As clinicians we have an assumption of what the "good practice" is in relation to the patients, but we have little insight, into the patients' own experience and satisfaction.

Objectives: To investigate the patients views on the level of information, and their experience and satisfaction with the doctor and nurse-led consultations in the outpatient clinic.

Methods: The approach of the survey was both quantitative and qualitative. A questionnaire was designed with four questions on a matrix scale concerning their information level on their disease/symptoms; their treatment; if they had received written material and if so, it's value; and their possibility to talk about subjects that matters for them during consultations. To gain a qualitative approach with focus on the patients' experiences and comments, every question was constructed with space for comments. Comments were transcribed, indexed, analysed, and thematically divided. The inclusion of patients were all patients visiting the clinic within a specific week in January 2019.
Results: The results from the four questions, are shown in the figures below, measured in percentage. In total, 283 participated (response rate 60\%).
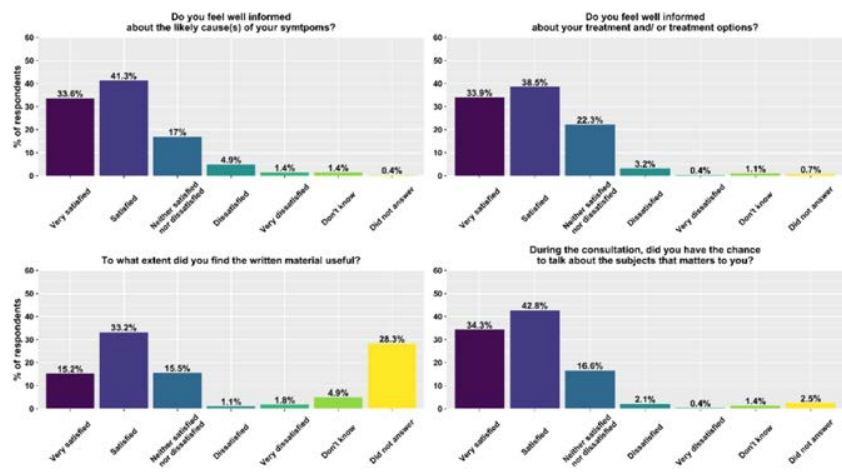

Four themes covered from the analysis of patients' comments. "Seeking own answers", "Own coping - effort", "Conversation with doctor/nurse" and "Treatment/medication". Comments revealed many descriptions and stories of patients showered with all types of suggestions of taking control or managing the disease by them self, from their family or media. It also revealed their interest in discussing, these subjects and matters with the doctor/nurse, without being rejected. The feeling of rejection resulted in some patients trying other treatments or introducing changes in their lifestyle, without involving the doctor. The patients also called for more knowledge about physical training, and not only medical treatment.

Conclusion: In general, the patients had a very positive perception towards the consultations, and the information from the outpatient clinic. Despite that, the themes indicated a pattern, that needs to be considered, so clinicians acknowledge the patients wish for guidance, besides the medical treatment. To support the patient, without leaving them with a feeling of being rejected, we need to consider how to articulate the subjects the patients are exposed to outside our clinic, so the patients feel free to inform or involve the clinic instead of being silent, in risk of counteracting the medical treatment.

Acknowledgments: We are grateful to the participants who shared their experiences. We also thank an internal research group taking part of the investigation. Disclosure of Interests: Ida Lund: None declared, Annette Hansen Consultant of: AbbVie, Speakers bureau: Eli Lily, Betina Stampe: None declared, René Cordtz: None declared, Lene Dreyer: None declared

DOI: 10.1136/annrheumdis-2020-eular.422

\section{SAT0633-HPR AN EXPLORATION INTOTHE CONVERSATIONS AROUND SEXUAL FUNCTIONING THAT MALES WITH SJÖGREN'S SYNDROME HAVE ON AN INTERNET FORUM.}

J. Mccready ${ }^{1}$, K. Mccarty ${ }^{2}$, V. Deary ${ }^{2,3}$, T. Collins ${ }^{1}$, K. Hackett ${ }^{1,3} .{ }^{1}$ Northumbria University, Department of Social Work, Education, and Community Wellbeing, Newcastle upon Tyne, United Kingdom; ${ }^{2}$ Northumbria University, Department of Psychology, Newcastle upon Tyne, United Kingdom; ${ }^{3}$ Newcastle upon Tyne Hospitals NHS Foundation Trust, Newcastle upon Tyne, United Kingdom

Background: Sjögren's Syndrome (SS) is an autoimmune rheumatic disease that targets secretion glands throughout the body, causing symptoms of oral ocular and genital dryness (van de Merwe, 2010). A small body of literature has investigated the impact of SS on women's sexual functioning, however, no research currently exists that has explored this topic in males with SS. Gathering a snapshot of issues may prove difficult given the sensitivity of the topic Therefore, data must be gathered from sources where individuals may have the confidence to talk openly. Research has shown that many patient groups turn to internet forums to discuss sensitive issues under the cloak of anonymity (White \& Dorman, 2001). Analysing this source of data allows us to explore the conversations pertaining to sexual functioning that males with SS may not feel comfortable discussing in a traditional qualitative setting, and may be instrumental in guiding future intervention strategies.

Objectives: To explore the conversations around sexual functioning that male users with SS have on an internet forum.

Methods: A large publicly accessible internet forum that individuals with a diagnosis of SS used to discuss issues and share experiences with other users was selected. Thread names and post content were scraped using a web scraping tool and posts identified as containing relevant keywords were exported into Excel. Braun \& Clarke's (2006) thematic analysis was used to analyse post content. Results: A total of 78 posts were identified as being pertinent to the topic of male sexual functioning. Conversations were predominately centred on symptom presence in the reproductive organs. Forum users discussed having fluctuating pain in the testicles, scrotum, groin, anus, and rectum. They also reported experiencing 
feelings of dryness at the base of the penis, around the testicles, under the foreskin, around the glans of the penis and in the anus. Discussions were also had about changes in the volume and consistency of seminal fluid released either prior to or during ejaculation. Another conversation theme revolved around how the symptoms they experienced affected their ability to engage in sexual intercourse. Forum users discussed how pain and dryness made sexual intercourse painful, resulting in them withdrawing from sexual activity indefinitely. Discussions were also had about the lack of information available to help understand and manage sexual dysfunction. Forum users discussed how feelings of embarrassment about the nature of the symptoms and the stigma of it being "a woman's disease" kept them from seeking medical assistance. Those who had sought medical assistance shared their belief that health professionals (HPs) were misdiagnosing their symptoms and were prescribing ineffective treatments. They also reported that their HPs were dismissive of symptoms and unwilling to assist further. Conclusion: Utilising conversations from an internet forum was an effective method to use to gain insight into some of the issues that males with SS experience with sexual functioning. The absence of accessible information and lack of support from HPs for males with SS is hinted at in this research. Future research should focus on identifying issues surrounding male sexual functioning as this will both guide future intervention strategies and allow HPs to publish material to better support males with SS. References:

[1] Braun, V., \& Clarke, V. (2006). Using thematic analysis in psychology. Qualitative Research in Psychology, 3(2), 77-101.

[2] van de Merwe, J. P. (2010). Sjögren's Syndrome: Information for patients and professionals. Rotterdam: Erasmus MC.

[3] White, M., \& Dorman, S. M. (2001). Receiving social support online: Implications for health education. Health Education Research, 16(6), 693-707.

Disclosure of Interests: None declared

DOI: 10.1136/annrheumdis-2020-eular.898

\section{SAT0634-HPR PATIENT-REPORTED OUTCOMES REGARDING TWO FORMS OF METHOTREXATE AUTOINJECTORS IN RHEUMATOID ARTHRITIS: AN INTERNATIONAL CROSS-OVER SURVEY.}

Y. Morvan ${ }^{1}$, J. D. Zeitoun ${ }^{2} .{ }^{1}$ Ipsos Healthcare, Paris, France; ${ }^{2}$ Saint-Antoine Hospital Assistance Publique Hôpitaux de Paris, Gastroenterology and Nutrition, Paris, France

Background: Several types of methotrexate (MTX) autoinjectors (AI) are currently marketed in rheumatoid arthritis (RA), yet comparative data are scarce. Objectives: Investigate respective perceptions of patients regarding two marketed forms of MTX Al via a survey conducted by a global market research company.

Methods: Patients with moderate to severe RA treated by one of the two forms of MTX Al were recruited. In each participating country (France, Ireland, United-Kingdom, Spain), the respective proportions of recruited patients were approximately aligned on local market shares. The two investigated devices were: A-Al/ The first MTX Al marketed in Europe: bigger size, with an activation button, without double injection sound-control, with a larger window; B-Al/ The second MTX Al commercialized in Europe: smaller and thinner size, without activation button, with double injection sound-control and a smaller window. Each patient was interviewed during 30 minutes on his or her satisfaction level with the currently used device. Then, they were presented the alternative Al and they could test it on skin-mimicking pads. After this step, the patients were interviewed on the alternative device.

Results: 100 patients were enrolled over one-month period (A-Al users, $n=65$; $B-A$ I users, $n=35$ ). Overall, $61 \%$ of $A$-Al users reported that B-Al was "better" or "much better" whereas $43 \%$ of B-Al users judged A-Al as "better" or "much better." When $\mathrm{B}-\mathrm{Al}$ users were asked to evaluate convenience elements of $\mathrm{A}-\mathrm{Al}$, recognition of injection ending, general design and ease of use were the indicators that were the most poorly judged $(60 \%, 54 \%$, and $46 \%$ respectively). When A-Al users were cross-tested for B-Al, injection mode, general feeling, and ease of use were the three items providing the greatest satisfaction $(80 \%, 77 \%$, and $75 \%$, respectively). When they were asked about the characteristics of their usual device, the button, the design of the device and discomfort associated with the injection were the most dissatisfactory elements $(30 \%, 31 \%, 34 \%$ respectively). Also, $73 \%$ of $\mathrm{A}-\mathrm{Al}$ users reported being interested in trying B-Al while $26 \%$ of B-Al users replied being so. Last, $95 \%$ of B-Al users declared being "very satisfied" or "totally satisfied", with ease of use and recognition of injection ending being the most attractive items ( $94 \%$ and $95 \%$ of high or full satisfaction respectively).

Conclusion: In this international cross-over survey, the newest autoinjector on the market, B-Al has shown to exhibit better reported outcomes with respect to ease of use and recognition of the end of the injection and other tested indicators.

Treatment of patients with RA should aim at the best care and must be based on a shared decision between the patient and the rheumatologist. ${ }^{1}$ In this scope, the shared decision making is permitted when the patient agrees with the choice of treatment (medication, administration route, device, etc.). Patient's involvement in decision making is assumed to lead to improvement in health outcomes such as a better adherence to the treatment. ${ }^{2}$

\section{References:}

[1] Smolen JS, Landewé R, Bijlsma J, et al EULAR recommendations for the management of rheumatoid arthritis with synthetic and biological disease-modifying antirheumatic drugs: 2016 update Annals of the Rheumatic Diseases 2017;76:960-977

[2] Nota,I.; Drossaert, C.H.; Taal,E.; Vonkeman, H.E.; van de Laar, M.A. Patient participation in decisions about disease modifying anti-rheumatic drugs: A cross-sectional survey. BMC Musculoskelet. Disord. 2014, 15, 333.

Disclosure of Interests: None declared

DOI: 10.1136/annrheumdis-2020-eular.3669

\section{SAT0635-HPR THE BURDEN OF SCLERODERMA FROM PATIENTS'} PERSPECTIVE

A. Opinc ${ }^{1}$, O. Brzezińska ${ }^{1}$, J. Makowska ${ }^{1} .{ }^{1}$ Medical University of Lodz, Department of Rheumatology, Łódź, Poland

Background: Scleroderma can significantly impair daily functioning by leading to multi-organ involvement and profound fatigue. Recent studies suggest that objective measures of the disease severity may not necessarily correspond with the patients' perception ${ }^{1}$.

Objectives: To evaluate the degree of disability and fatigue in patients with scleroderma, distinguish the most troublesome activities and assess which clinical symptoms had mostly impact on daily functioning.

Methods: An online questionnaire was created and distributed to English-speaking patients with scleroderma by means of online support groups. The questionnaire contained inquiries about demographical data, clinical symptoms as well as the Scleroderma Health Assessment Questionnaire (SHAQ, comprised of HAQ-DI and specific VAS scales) and Functional Assessment of Chronic Illness Therapy - Fatigue (FACIT-F). Standard and Alternative Disability Indexes (SDI and ADI) were counted based on answers in HAQ-DI part. Degrees of disability and fatigue, reflected by SDI, $\mathrm{ADI}$ and FACIT-F scores, were compared between subgroup of patients with and without particular clinical symptoms. Responses were analysed statistically.

Results: Questionnaire was completed by 85 responders (mean age $48,93 \pm 14,4$ $98,82 \%$ were female). Mean SDI was $1.09 \pm 0.66$ with $41.18 \%$ of patients fulfilling the criteria of mild to moderate difficulty $(\mathrm{SDI}<1), 50.59 \%$ classified as moderate to severe disability $(1 \leq \mathrm{SDI}<2)$ and $8.24 \%$ being severely or very severely disabled $(\mathrm{SDI} \geq 2)$. Mean value of $\mathrm{ADI}$ was $0.93 \pm 0.61 .56 .47 \%$ of responders needed aid of the caregivers while gripping/opening, $31.76 \%$ while reaching and $29.41 \%$ while running errands and chores. Most frequently used facilitating devices included devices for gripping/opening (32.94\%) and for maintaining hygiene (21.18\%). Mean value of overall disease severity was estimated by VAS as $52.06 \pm 0.66$. The mean values of VAS assessing the impact on daily living were respectively $48.16 \pm 29.28$ for pain related to the disease, $43.34 \pm 34.94$ for Raynaud's, $41.86 \pm 34.59$ for gastrointestinal problems, $31.60 \pm 31.38$ for breathing difficulties and $20.85 \pm 33.47$ for finger ulcers. Mean FACIT-F score was $23.62 \pm 11.88$ yet $71.76 \%$ of patients fulfilled the criteria of severe fatigue. Both SDI and ADI correlated with FACIT-F score (respectively $r=-0.5684, p<0.0001$ and $r=-0.5711, p<0.0001)$. SDI, ADI and FACIT-F correlated with VAS scores for overall disease severity, pain, gastrointestinal and breathing problems. Patients with symptoms such as arthralgia, muscle weakness, dysphagia, blurred vision, hair loss, unintentional weight loss and hoarseness were statistically more disabled and fatigued as compared to patients without such symptoms. Responders with skin ulcers/non-healing wounds (other than finger ulcers), Raynaud's or erythema/rash were more disabled than responders without such symptoms, yet no differences were observed in the degree of fatigue.

Conclusion: Majority of patients with scleroderma are moderately to severely disabled yet cope with the impairments with the widespread use of facilitat ing devices or aid of caregivers. Profound fatigue, observed in the majority of patients, may contribute to the high overall perception of disease severity. References:

[1] Jaeger VK, Distler O, Mauer B et al. Functional disability and its predictors in systemic sclerosis: a study from the DeSScipher project within the EUSTAR group. Rheumatology (Oxford). 2018 Mar 1;57(3):441-450. doi: 10.1093/ rheumatology/kex182

Disclosure of Interests: None declared

DOI: 10.1136/annrheumdis-2020-eular.3797

\section{SAT0636-HPR PATIENT EXPERIENCE WITH THE PRESCRIPTION, INFORMATION AND USE OF METHOTREXATE}

T. Oton $^{1}$, L. Carmona ${ }^{1}$, J. L. Andréu Sánchez ${ }^{2} .{ }^{1}$ InMusc. Instituto de Salud Musculoesquelética, Madrid, Spain; ${ }^{2}$ Rheumatology Department. Hospital Universitario Puerta de Hierro-Majadahonda, Majadahonda, Spain

Background: Methotrexate (MTX) is currently a mainstream drug in the treatment of rheumatic diseases. However, the response to MTX is not universal and 